\title{
Extension of a Modular Particle-Continuum Method to Vibrationally Excited, Hypersonic Flows
}

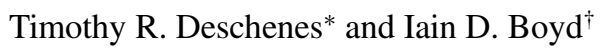 \\ University of Michigan, Ann Arbor, Michigan 48109
}

DOI: $10.2514 / 1 . J 050908$

\begin{abstract}
A modular particle-continuum method is extended to include vibrationally excited energy modes to simulate hypersonic steady-state flows that exhibit small regions of translational nonequilibrium in a mainly continuum flowfield. This method loosely couples an existing direct simulation Monte Carlo code to a Navier-Stokes solver (computational fluid dynamics) while allowing both time step and cell size to be completely decoupled between each method. A new information-transfer scheme that controls the inherently large statistical scatter of vibrational energies in low-temperature regions is described and tested. Two vibrational-relaxation models are implemented to test the sensitivity in agreement between direct simulation Monte Carlo and the modular particle-continuum method. By limiting the size of the direct simulation Monte Carlo region to only areas in translational nonequilibrium and maintaining consistent physical models in both computational fluid dynamics and direct simulation Monte Carlo modules, the modular particle-continuum method is able to reproduce full direct simulation Monte Carlo results for flow with global Knudsen number of 0.01 while decreasing the computational time required by a factor of about four.
\end{abstract}

\section{Nomenclature}

$C_{h}$

d

$=$ heat transfer coefficient

$=$ diameter, $\mathrm{m}$

$=$ energy per unit volume, $\mathrm{J} / \mathrm{m}^{3}$

$=$ probability density function

$=$ relative speed, $\mathrm{m} / \mathrm{s}$

$=$ vibrational energy level

$=$ molecular mass, $\mathrm{kg}$

$=$ number density, $1 / \mathrm{m}^{3}$

$=$ average probability

$=$ pressure, $\mathrm{Pa}$

$=$ flow quantity

$=$ heat flux, $\mathrm{W} / \mathrm{m}^{2}$

$=$ internal relaxation source term

$=$ temperature, $\mathrm{K}$

$=$ macroscopic velocity, $\mathrm{m} / \mathrm{s}$

$=$ macroscopic speed, $\mathrm{m} / \mathrm{s}$

$=$ source term, $\mathrm{J} / \mathrm{s}$

$=$ collision number vibrational temperature

$=$ characteristic temperature, $\mathrm{K}$

$=$ mean free path, $\mathrm{m}$

$=$ mean collision frequency, $1 / \mathrm{s}$

$=$ mass density, $\mathrm{kg} / \mathrm{m}^{3}$

$=$ reference cross section, $\mathrm{m}^{2}$

$=$ relaxation time, $\mathrm{s}$

$=$ subrelaxation weight

$=$ variable probability
$=$ Boltzmann constant, $1.38 \times 10^{-23} \mathrm{~J} / \mathrm{K}$

$=$ ratio of vibrational temperature to characteristic

$=$ number of internal degrees of freedom

$=$ coefficient of viscosity, $\mathrm{kg} / \mathrm{ms}$, reduced mass, $\mathrm{kg}$

Presented at the Aerospace Sciences Meeting, Orlando, FL, 5-8 January 2009; received 8 September 2010; revision received 5 April 2011; accepted for publication 29 April 2011. Copyright $\odot 2011$ by Timothy R. Deschenes and Iain D. Boyd. Published by the American Institute of Aeronautics and Astronautics, Inc., with permission. Copies of this paper may be made for personal or internal use, on condition that the copier pay the $\$ 10.00$ per-copy fee to the Copyright Clearance Center, Inc., 222 Rosewood Drive, Danvers, MA 01923; include the code 0001-1452/11 and \$10.00 in correspondence with the CCC.

*Graduate Student, Department of Aerospace Engineering; thytimo@ umich.edu. Student Member AIAA.

'Professor, Department of Aerospace Engineering; iainboyd@umich.edu. Associate Fellow AIAA. $\omega=$ variable-hard-sphere viscosity-temperature exponent

$\begin{array}{ll}\text { Subscripts } & \\ \text { CFD } & =\text { computational fluid dynamics } \\ \text { DSMC } & =\text { direct simulation Monte Carlo } \\ \text { MW } & =\text { Millikan and White } \\ P & =\text { Park } \\ \text { ref } & =\text { reference } \\ \text { ROT } & =\text { rotational } \\ S & =\text { species } \\ \text { TRA } & =\text { translational } \\ \text { VIB } & =\text { vibrational } \\ \infty & =\text { freestream }\end{array}$

\section{Introduction}

W ITH the increased interest in hypersonic vehicles, for scientific, defense, and commercial uses, there is a need for efficient and accurate computation of the flowfield, aerodynamics, and heat transfer. Throughout the flight regime, hypersonic vehicles experience flowfields that can range from being completely rarefied to continuum. At very high altitudes, the flow is entirely rarefied or in translational nonequilibrium, whereas at low altitudes, the flowfield could be considered thoroughly continuum. Mature methods exist that are capable of computing rarefied or continuum flows. The direct simulation Monte Carlo (DSMC) method is often used to simulate highly rarefied flow conditions [1]. Although accurate over the entire flow regime, the computational expense of performing DSMC on high-density flowfields becomes increasingly expensive. In only the continuum regime, the Navier-Stokes equations are applicable and can be solved accurately and efficiently using modern computational fluid dynamics (CFD). In addition, models are available for both methods to account for excited vibrational modes and chemical reactions that can become important in high-enthalpy flows. At intermediate altitudes, a flow can be characterized as mainly continuum with localized areas, such as the shock, boundary layer, and wake, that display translational nonequilibrium effects. Because of the local rarefied regions, the Navier-Stokes equations are not accurate. Although DSMC is accurate over the entire flowfield, it is expensive for low-altitude simulations that still contain small regions of flow that are rarefied. Instead, a hybrid code can be used that solves the flowfield with CFD in the continuum region and uses DSMC in the locally rarefied regions. Speedup can be achieved, since restrictions imposed by DSMC (specifically, the collisional cell size 
must remain smaller than the mean free path and the time step must remain less than the mean free time) are not required for CFD calculations, so decoupling of both mesh densities and time step are achievable. This method can take advantage of the computational speed up of CFD in regions where it is applicable, while still maintaining the physical accuracy of DSMC in the regions where the Navier-Stokes equations break down.

Previous work has been performed using a "zonally decoupled" DSMC-CFD simulation [2-4]. For these hybrid methods, a CFD solution is calculated to a predetermined interface, then this information is used as the boundary condition for the DSMC simulation over the remainder of the domain. This method is only valid when the rarefied region is completely down stream of the continuum region with no recirculation occurring across the interface. This is because the CFD solution is completely decoupled from the DSMC solution. Often, rarefied regions are highly localized and two way coupling is required to obtain an efficient, physically accurate simulation. Various methods have been proposed that adaptively reposition the interface between DSMC and CFD throughout the simulation using different coupling methods between the two domains [5-8]. Reference [9] presents a discussion of the major considerations involved in coupling a kinetic scheme with a continuum method as well as a summary of published work on past methods.

The present paper extends the modular particle-continuum (MPC) method that was first developed for 1-D shock waves [9] and later extended to 2-D and axisymmetric flows [10,11]. In previous work, the MPC method was able to reproduce full DSMC results for macroscopic flowfield quantities, surface properties, and velocity probability density functions. By limiting the DSMC method to only regions that are rarefied, the MPC method can achieve speedup factors exceeding 3 for transitional flows [12] and up to 28 for nearcontinuum flows $[11,13]$. In addition, previous work was performed to increase the consistency between flow modules by inclusion of similar rotational relaxation models in both CFD and DSMC [13].

This paper outlines the extension of the method by including vibrational excitation within both the CFD and DSMC modules of the MPC method. This is required to maintain the physical accuracy needed to simulate high-enthalpy flows. Two different vibrationalrelaxation methods are used within the DSMC method. Assessment of agreement between the MPC method and full DSMC using each vibrational method is performed. Heat transfer and computational speedup are presented for simulations employing different physical models in the flow modules. Finally, conclusions are formulated based on the current results.

\section{Hybrid Method}

The MPC method [14] used in this study was developed to be capable of simulating one-dimensional shock waves [9] and both axisymmetric and two-dimensional, steady-state, hypersonic flows $[10,14]$. It uses the CFD code LeMANS $[15,16]$ for the continuum regions and uses the DSMC code MONACO [17] to simulate the rarefied regions.

\section{A. Flow Modules}

LeMANS is capable of simulating 2-D, axisymmetric, or 3-D steady-state, continuum, laminar, hypersonic flows using a finite volume formulation of the Navier-Stokes equations with the added ability to account for vibrational and rotational nonequilibrium. The inviscid fluxes are solved using a less dissipative, modified form of the Steger-Warming flux vector splitting method that can be used to calculate boundary layers [16]. The method switches back to the original form of Steger-Warming near shock waves. The viscous terms are calculated using properties at cell nodes and cell centers. The no-slip condition is applied to wall boundaries, and the inflow and outflow are treated as supersonic flow. Time integration is performed using a point-implicit method. It can also simulate multispecies and weakly ionized flows with finite rate chemistry, but those models are not used in this study. A separate rotational energy equation is solved and rotational-translational relaxation is modeled using the rotational collision number of Parker [18] as described in
[19]. The variable-hard-sphere (VHS) model is used to calculate the viscosity-temperature relation that can be formulated using Eqs. (1) and (2), where $\omega, \mu_{\text {ref }}$, and $T_{\text {ref }}$ are constants and $d_{\text {ref }}$ is the species reference diameter [1]. All results presented within this paper simulate molecular nitrogen with a reference diameter of $d_{\text {ref }}=$ $4.17 \times 10^{-10} \mathrm{~m}$ at $T_{\text {ref }}=273 \mathrm{~K}$ with a power law exponent of $\omega=0.75$ :

$$
\begin{gathered}
\mu=\mu_{\text {ref }}\left(\frac{T_{\mathrm{TRA}}}{T_{\text {ref }}}\right)^{\omega} \\
\mu_{\text {ref }}=\frac{15 \sqrt{\pi m k T_{\text {ref }}}}{2 \pi d_{\text {ref }}^{2}(5-2 \omega)(7-2 \omega)}
\end{gathered}
$$

MONACO is a general cell-based implementation of the DSMC method capable of simulating rotational and vibrational nonequilibrium and multispecies flow with finite rate chemistry. The VHS collision model that replicates the macroscopic viscositytemperature dependence modeled in LeMANS is used. Rotationaltranslational relaxation is modeled using the variable rotational energy exchange probability of Boyd [20], which is also based on Parker [18]. The proceeding subsections describe and compare the vibrational-relaxation process modeled within both the CFD and DSMC modules.

\section{B. Vibrational Relaxation}

Both MONACO and LeMANS have the capability of simulating vibrational nonequilibrium. For the CFD module, a separate vibrational energy equation is added to the total system of equations solved. Equation (3) shows the time rate of change of vibrational energy per unit volume, where $\mathbf{q}_{\mathrm{vIB}}$ is the vibrational heat flux given by Fourier's law, $\mathbf{u}_{d s}$ is the species diffusion velocity given by Fick's law, and $\dot{w}_{\text {VIB }}$ is the vibrational source term:

$$
\begin{array}{cc}
\frac{\partial E_{\mathrm{VIB}}}{\partial t}+\nabla \cdot\left(E_{\mathrm{VIB}} \mathbf{u}\right)=-\nabla \cdot\left(\mathbf{q}_{\mathrm{VIB}}\right)-\nabla \cdot \sum_{s}\left(\rho_{s} e_{\mathrm{VIB}, s} \mathbf{u}_{d s}\right) \\
+\dot{w}_{\mathrm{VIB}} \\
\dot{w}_{\mathrm{VIB}}=\sum_{s}\left(S_{\mathrm{VIB}, s}^{\mathrm{TR}-\mathrm{VIB}}+\dot{w}_{s} e_{\mathrm{VIB}, s}\right)
\end{array}
$$

The vibrational source term can be split into two parts: a vibrational-translational relaxation portion and a source due to the gain and loss of vibrational energy through chemical reactions as shown in Eq. (4). For this work the chemistry source term is always zero. The vibration-translation relaxation source term can be written as Eq. (ㄷ), where $e_{\mathrm{VIB}, s}^{*}$ is the specific vibrational energy evaluated at the translational temperature and $\tau_{\mathrm{VIB}, s}$ is the species vibrationtranslation relaxation time. This relaxation time can be calculated using the summation of the Millikan and White correlation [21] with Park's high-temperature correction [22]. Equations (7) and (8) respectively show the relaxation times using Millikan and White correlation [21] and Park's high-temperature correction [22], where $A_{s}$ and $B_{s}$ are constants given by Millikan and White correlations [21], $p$ is the pressure, $\sigma_{s}$ is the reference species collision cross section, $n$ is the total number density, and $m_{s}$ is the species mass. For this work, a reference collision cross section of $5.81 \times 10^{-21} \mathrm{~m}^{2}$ is used:

$$
\begin{gathered}
S_{\mathrm{VIB}, s}^{\mathrm{TRA}-\mathrm{VIB}}=\rho_{s} \frac{e_{\mathrm{VIB}, s}^{*}-e_{\mathrm{VIB}, s}}{\tau_{\mathrm{VIB}, s}} \\
\tau_{\mathrm{VIB}, s}^{\mathrm{CFD}}=\tau_{\mathrm{MW}, s}+\tau_{P, s} \\
\tau_{\mathrm{MW}, s}=\frac{1}{p} \exp \left[A_{s}\left(T_{\mathrm{TRA}}^{-\frac{1}{3}}-B_{s}\right)-18.42\right]
\end{gathered}
$$




$$
\tau_{P, s}=\frac{\sqrt{\frac{\pi m_{s}}{8 k T_{\mathrm{TRA}}}}}{\sigma_{s} n}
$$

The DSMC module has two different models available to simulate the vibration-translation relaxation process. The first is a phenomenological model based on the method described by Boyd [23] that calculates a variable probability of a vibrationally inelastic collision occurring based on the relative velocity between two colliding particles. First, separate variable probabilities for the Millikan and White relaxation time and the Park high-temperature time are formed. The form of the Millikan and White variable probability is given by Eq. (9), where $Z_{o}, \alpha$ and $g^{*}$ are found from VHS parameters and by evaluating Eq. (10) such that the relaxation process reproduces macroscopic Millikan and White relaxation times under continuum conditions. The evaluation of the integral is approximated using the method of steepest descent. In a similar manner, an instantaneous probability can be formulated for Park's high-temperature correction such that it satisfies Eq. (11). The total probability for a vibrationally inelastic collision can be formulated from the two variable probabilities as shown in Eq. (12). To account for the differences in the definition of relaxation times between DSMC and CFD, the Gimelshein et al. [24] correction factor, shown in Eq. (13), is applied to all DSMC vibrational-relaxation times:

$$
\begin{gathered}
\phi_{\mathrm{MW}}(g)=\frac{1}{Z_{o}} g^{\alpha} \exp \left(\frac{-g^{*}}{g}\right) \\
\frac{1}{\tau_{\mathrm{MW}} \nu_{s}}=\int_{0}^{\infty} \phi_{\mathrm{MW}}(g) f(g) \mathrm{d} g \\
\frac{1}{\tau_{P} \nu_{s}}=\int_{0}^{\infty} \phi_{P}(g) f(g) \mathrm{d} g \\
\phi_{\mathrm{tot}}=\frac{\phi_{\mathrm{MW}} \phi_{P}}{\phi_{\mathrm{MW}}+\phi_{P}} \\
\tau_{\mathrm{TRA}-\mathrm{VIB}}^{\mathrm{DSMC}}=\tau_{\mathrm{TRA}-\mathrm{VIB}}^{\mathrm{CFD}} \frac{\eta_{\mathrm{TRA}}}{\eta_{\mathrm{TRA}}+\frac{1}{2} \eta_{\mathrm{VIB}, \mathrm{TRA}}^{2} \exp \left(\frac{\theta_{\mathrm{VIB}}}{T_{\mathrm{TRA}}}\right)}
\end{gathered}
$$

Approximations to evaluation of the integral in Eq. (10) can cause discrepancy in relaxation times predicted by CFD and DSMC in regions of the flow that can be considered continuum $[19,25,26]$.

To test the effect of these discrepancies on the agreement of the MPC method with full DSMC, a second, cell-based vibrationalrelaxation model is implemented as well. This method uses the macroscopic translational temperature to calculate the continuum relaxation time using Eq. (6) and applies the Gimelshein correction factor to find the DSMC relaxation time. The average probability within the cell is then calculated using Eq. (14) and applied to all collisions in the cell:

$$
\langle P\rangle_{\mathrm{VIB}}=\frac{1}{\tau_{\mathrm{VIB}, s}^{\mathrm{SSMC}} v_{s}}
$$

Regardless of the method of calculating the probability of a vibrationally inelastic collision, both methods use a LarsenBorgnakke procedure [27] for discrete energy levels to model the transfer between available translational and vibrational energy modes. Figure 1 shows a comparison of an adiabatic vibration relaxation process using the two DSMC methods and a continuum calculation. The cell-based vibrational-relaxation rate is in much better agreement with the continuum calculation than the variable vibrational-relaxation probability. The effect of the improved agreement in prediction of vibrational relaxation between DSMC

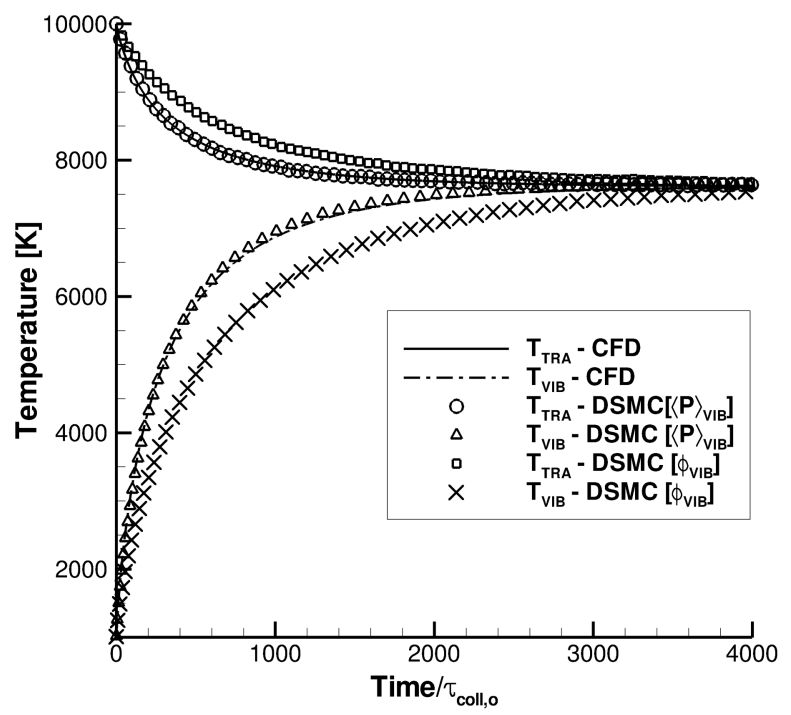

Fig. 1 Comparison of adiabatic vibrational-translational relaxation process predicted by DSMC and CFD.

and CFD methods in continuum regions will be tested within a hybrid DSMC-CFD code in the proceeding sections.

\section{Interface Location and Continuum Breakdown}

Both the accuracy and efficiency of a hybrid DSMC-CFD method depend strongly on proper placement of the interface location. For physical accuracy, the interface location must be located within regions that can be considered in translational equilibrium, where the velocity distribution is only slightly perturbed from equilibrium, and the Navier-Stokes equations are valid. The efficiency of the hybrid method requires the interface between CFD and DSMC to be located near the edge of the rarefied region. The MPC method uses a gradient-length Knudsen number first proposed by Boyd et al. [28] and Wang and Boyd [29]. Equation (15) shows the gradient-length Knudsen number, where $\lambda$ is the local mean free path and $Q$ is some flow quantity of interest. For this work, gradient-length Knudsen numbers based on density, velocity magnitude, translational temperature, and rotational temperature are used:

$$
K n_{G L-Q}=\lambda\left|\frac{\nabla Q}{Q}\right|
$$

Previous research has shown for hypersonic reentry problems $[10,30]$ and 1-D normal shock waves [9] that regions where the maximum gradient-length Knudsen number remains less than 0.05 display less than a $5 \%$ difference in flow quantities predicted by DSMC and CFD assuming perfect gas physics. Therefore, regions where the maximum gradient-length Knudsen number exceeds 0.05 are simulated with DSMC to maintain physical accuracy within the solution. In addition, Deschenes et al. [13] have found that an additional breakdown parameter, shown in Eq. (16), must be applied to ensure that the strong thermal relaxation process between rotational and translational energy modes is simulated with the DSMC method. Since vibrational-translational relaxation is much slower than rotational-translational relaxation, no additional breakdown parameter is needed with the addition of vibrational excitation. This results in a final breakdown parameter shown in Eq. (17):

$$
K n_{\mathrm{ROT}-\mathrm{NEQ}}=\frac{\left|T_{\mathrm{TRA}}-T_{\mathrm{ROT}}\right|}{2 T_{\mathrm{ROT}}}
$$

$B r=\max \left(K n_{G L-\rho}, K n_{G L-T_{\mathrm{TRA}}}, K n_{G L-T_{\mathrm{ROT}}}, K n_{G L-|V|}, K n_{\mathrm{ROT}-\mathrm{NEQ}}\right)$ 


\section{Information Transfer}

The modular implementation of the MPC method allows both the DSMC and CFD modules to maintain their own mesh and data structure. State-based coupling is performed, which assigns boundary conditions on the CFD domain from corresponding DSMC cells and vice versa and is implemented in a loosely coupled fashion such that information is not transferred after every iteration. Figure 2 shows a schematic of how data are transferred between the two modules at an interface location. First, the interface locations are determined using the breakdown parameter on a full CFD solution. Next, a DSMC buffer region is extended and cells that are used for boundary conditions are added to the end of the particle and continuum domains.

An unsteady period occurs in which the flowfield changes from the initial CFD solution to a hybrid particle-continuum result. During this time, the interfaces are allowed to move and macroscopic information is tracked in DSMC cells using a subrelaxation average proposed by Sun and Boyd [31], which is shown in Eq. (18), where $\langle Q\rangle_{j}$ is the average at the current iteration, $\langle Q\rangle_{j-1}$ is the average at the previous iteration, $Q_{j}$ is the value at the current iteration and $\Phi$ is the weighting used:

$$
\langle Q\rangle_{j}=(1-\Phi)\langle Q\rangle_{j-1}+\Phi Q_{j}
$$

A $\Phi$ value of 0.001 is used throughout all the MPC simulations described in this paper.

Although the subrelaxation technique reduces the scatter of macroscopic flow variables, it should only be used if the flow variables of interest are resolved in less than $\frac{1}{\Phi}$ iterations. For almost all flow variables, this can often be done in very few iterations. For vibrational energies, a discrete probability density function is used that is consistent with the simple harmonic oscillator assumption and the number of iterations required to resolve a low vibrational temperature becomes enormous. Figure $\underline{3}$ compares the subrelaxation average rotational and vibrational temperatures as a function of iteration number with 20 particles per iteration at various values of temperature. In general, the rotational temperature (and also translational temperature, which is not shown) can be resolved in very few iterations and a subrelaxation parameter of 0.001 is sufficient regardless of the mean energy content, since the discrete energy steps of these modes are much less than the mean particle energy content and the energy probability density functions can be considered continuous. In contrast, most freestream vibrational energies are much less than the discrete energy step size and the probability of experiencing a vibrationally excited molecule is extremely low. For example, when the vibrational temperature is less than $0.1 \theta_{\mathrm{VIB}}$ (which corresponds to a freestream temperature of less than $339.5 \mathrm{~K}$ for $N_{2}$ ), less than 2 vibrationally excited molecules are experienced in each set of $\frac{1}{\Phi}$ iterations. Decreasing the subrelaxation parameter, $\Phi$, could decrease the statistical scatter of the subrelaxation averaged vibrational temperature at the expense of efficiency, but typical highaltitude temperatures would require $\Phi$ to be many orders of magnitude smaller than what is currently used. This would make any coupled hybrid method using this technique slower than full DSMC.

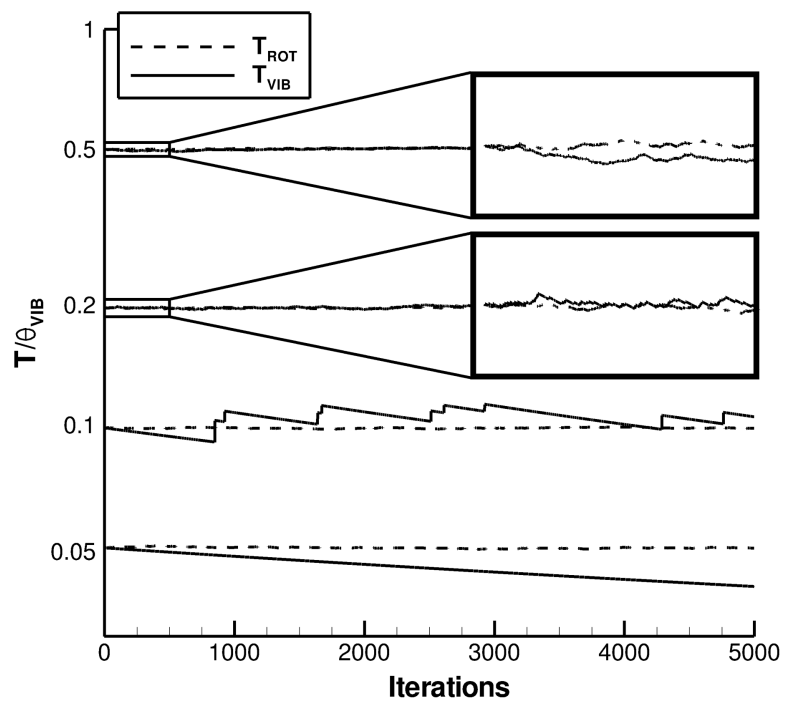

Fig. 3 Comparison of the level of statistical scatter of subrelaxation averages of internal temperatures at various levels.

Instead of assigning vibrational energies consistent with the discrete Boltzmann energy probability density function, the average vibrational energy is assigned to all particles in the boundary cells. Equation (19) shows the final calculation of the vibrational energy, where $N_{\max }$ is the level at which the discrete Boltzmann distribution is truncated, $R$ is the universal gas constant, $\varsigma$ is the ratio of vibrational temperature to characteristic vibrational temperature shown in Eq. (21), and $P_{i}$ is the probability of a particle having the $i$ th level of vibrational energy. Assuming vibrational energy is modeled as a simple harmonic oscillator, the probability of a particle being in a vibrational level can be calculated using Eq. (20). The maximum level, $N_{\max }$, is chosen such that the probability of a particle having a vibrational energy greater than that level is less than $1 \times 10^{-8}$ :

$$
\begin{gathered}
E_{\mathrm{VIB}}=\sum_{i=0}^{N_{\max }} P_{i} i \theta_{\mathrm{VIB}} R \\
P_{i}=\exp [-i / \varsigma](1-\exp [-1 / \varsigma]) \\
\varsigma=\frac{T_{\mathrm{VIB}}}{\theta_{\mathrm{VIB}}}
\end{gathered}
$$

At higher vibrational temperatures, such that $\varsigma>0.2$, vibrational energies can be sampled from the discrete Boltzmann probability density function without adversely affecting the efficiency of the MPC method and may be necessary for physical processes that directly depend on the vibrational energy distribution function such as chemistry. Based on the results shown in Fig. $\underline{3}$, a switching

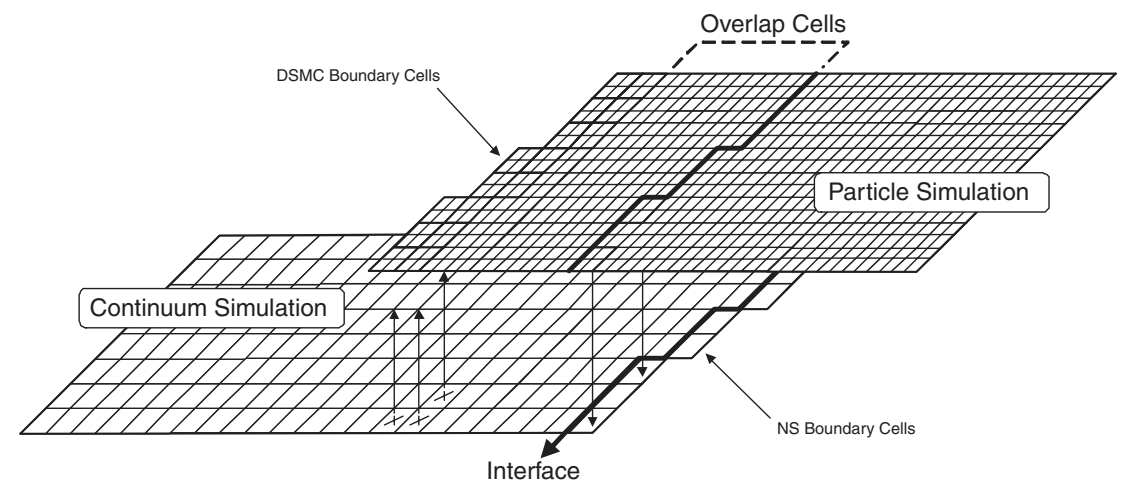

Fig. 2 State-based coupling [14]. 
parameter $\varsigma$ may be used to change from assigning average energies at low temperatures to sampling energies directly from the discrete Boltzmann energy probability density function at higher temperatures. The current results shown in Fig. 3 suggest that a switching value of 0.5 may be sufficient, such that the average value is assigned when $\varsigma<0.5$ and particle vibrational energies are sampled from the Boltzmann pdf when $\varsigma>0.5$. In this current work, the cell average vibrational energy is applied to all regions to evaluate the effect of the limiting case of setting the switching value to infinity. Regardless of the method used to assign vibrational energies, discrete vibrational levels are selected after vibrationally inelastic collisions. Since MONACO tracks a particle's vibrational energy mode based on the amount of energy, rather than the vibrational energy level, a mixture of average and discrete energy modes can exist in cells where only some boundary particles have experienced at least one vibrationally inelastic collision with no modifications to the DSMC module.

\section{Flow Conditions}

Hypersonic flow about a cylinder with a freestream Mach number of 15 is simulated. The freestream gas is $N_{2}$ with a number density of $n_{\infty}=1.61 \times 10^{21} \mathrm{~m}^{-3}$ and equilibrium temperatures set at $T_{\mathrm{TRA}, \infty}=T_{\mathrm{ROT}, \infty}=T_{\mathrm{VIB}, \infty}=217.5 \mathrm{~K}$. This corresponds to a freestream density of $\rho_{\infty}=7.48 \times 10^{-5} \mathrm{~kg} \mathrm{~m}^{-3}$ and a pressure of $p_{\infty}=4.22 \mathrm{~Pa}$. The diameter of the cylinder simulated is $d=8 \mathrm{~cm}$, which results in a global Knudsen number of $K n_{\infty}=0.01$. The cylinder wall temperature is set to $T_{w}=1000 \mathrm{~K}$ with full diffuse reflection in DSMC simulations and a no-slip isothermal condition in CFD simulations. The vibrational characteristic temperature used for all vibration temperature calculations is $\theta_{\text {vib }}=3395 \mathrm{~K}$. A constant time step of $1.5 \times 10^{-8} \mathrm{~s}$ is used in the DSMC method for all simulations, and a maximum Courant-Friedrichs-Lewy number of 50 is used in all CFD simulations. The full DSMC simulation requires about 25 million particles, and the full CFD calculation is performed on a mesh of 30,000 cells.

Case $\mathbf{M 1 5}_{\mathrm{VP}}$ corresponds to the case where vibrational relaxation in the DSMC module is simulated using the variable probability method based on the relative velocity, which is shown in Eq. (12). Case $\mathbf{M 1 5} \mathbf{C B}_{\mathbf{B}}$ corresponds to the case where vibrational relaxation in the DSMC module is simulated using the cell-based constant probability method, which is shown in Eq. (14).

\section{Numerical Results of Nonequilibrium Flows}

The purpose of the MPC method is to reproduce complete DSMC results to within $5 \%$ at a reduced computational expense. This section compares flowfield and surface-property results obtained with pure DSMC, full CFD, and MPC methods. In addition, code efficiency of the MPC method compared with full DSMC is discussed. Figure $\underline{4}$ shows the final and initial interface locations for the $\mathbf{M 1 5}_{\mathbf{C B}}$ case.
The interface locations for both cases are very similar, so only one is shown. Also, the extraction lines that are used to compare flow variables are indicated.

\section{A. Flowfield Properties}

Figures $5 \mathrm{a}$ and $5 \mathrm{~b}$ compare the translational and vibrational temperature contours predicted by DSMC, CFD, and MPC methods for the $\mathbf{M 1 5}_{\mathrm{VP}}$ case. For the prediction of translational temperatures contours, the MPC method is in much better agreement with DSMC than full CFD is with DSMC. The largest discrepancy observed between the MPC and DSMC results for any flow variable other than the vibrational temperature remains below $4 \%$. When comparing vibrational temperature contours, the MPC method greatly improves agreement with the full DSMC results. The worst agreement occurs in the expansion region where the MPC and full DSMC predicted vibrational temperatures deviate up to about $8 \%$. This could partially be attributed to the difference in relaxation rates computed in full DSMC and the CFD module of the MPC in this region.

Figure $6 \mathrm{a}$ shows a comparison of translational temperature contours predicted by full DSMC, full CFD, and MPC methods for the $\mathbf{M 1 5}_{\mathbf{C B}}$ case. Again, agreement between DSMC and the MPC predictions is excellent, whereas full CFD still fails to accurately predict nonequilibrium flowfield features. The largest discrepancy found between DSMC and the MPC results remains below $5 \%$ for all flow variables. Figure $6 \mathrm{~b}$ compares the vibrational temperature contours predicted by full DSMC, CFD, and MPC methods. Again, the MPC method can accurately reproduce the full DSMC solution even in areas where full DSMC and full CFD are in very poor agreement. Still, there is a discrepancy between the MPC method and full DSMC in the expansion region, but the agreement between full DSMC and MPC methods has improved with the maximum difference between DSMC and the MPC result remaining below 4\%. This is directly due to the improved agreement in the modeling of vibrational-relaxation rates between both flow modules in continuum regions.

Figure 7 compares the temperature predictions along a $45^{\circ}$ extraction line by full DSMC, full CFD, and MPC methods with each vibrational-relaxation model. In general, the MPC method has significantly improved agreement with DSMC compared with CFD regardless of the relaxation model. Even in regions that are considered continuum, and the CFD module is used, the MPC method can provide near perfect agreement with full DSMC due to the improved boundary conditions provided to the CFD solver. Though the cell-based relaxation model provides slightly improved agreement with full DSMC for vibrational temperature at around $x=0.04 \mathrm{~m}$, the difference in the two relaxation models is negligible along this extraction line.

Figures $8 \mathrm{a}$ and $8 \mathrm{~b}$ show the vibrational temperature predicted by DSMC, $\mathrm{CFD}$, and $\overline{\mathrm{MPC}}$ methods along the $135^{\circ}$ extraction line for

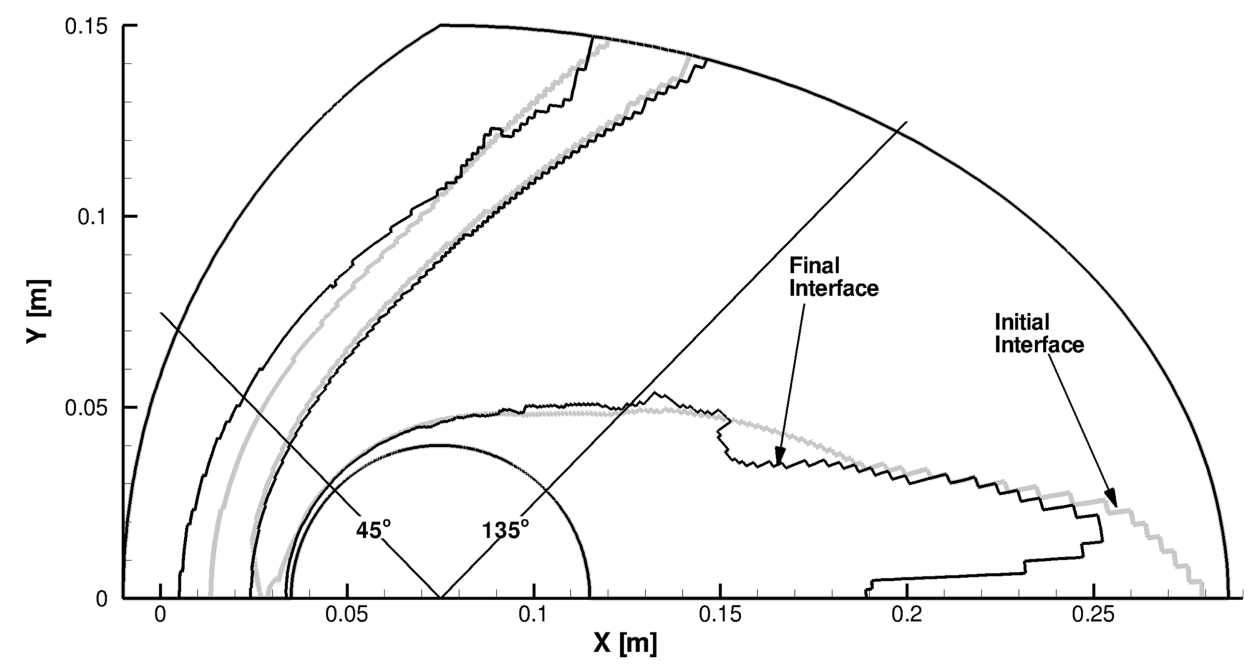

Fig. 4 Initial and final DSMC-CFD interface locations for the $M 15_{C B}$ case. 


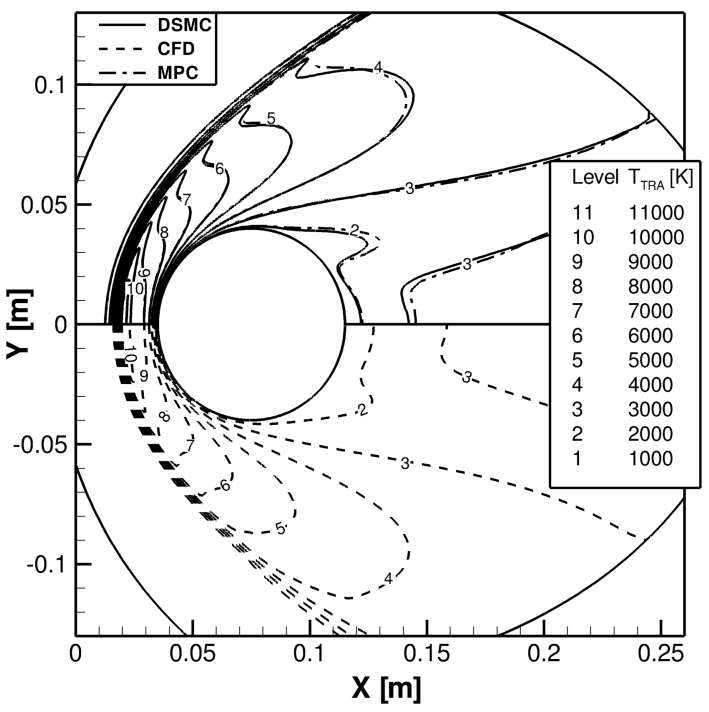

a) $\boldsymbol{T}_{\text {TRA }}$

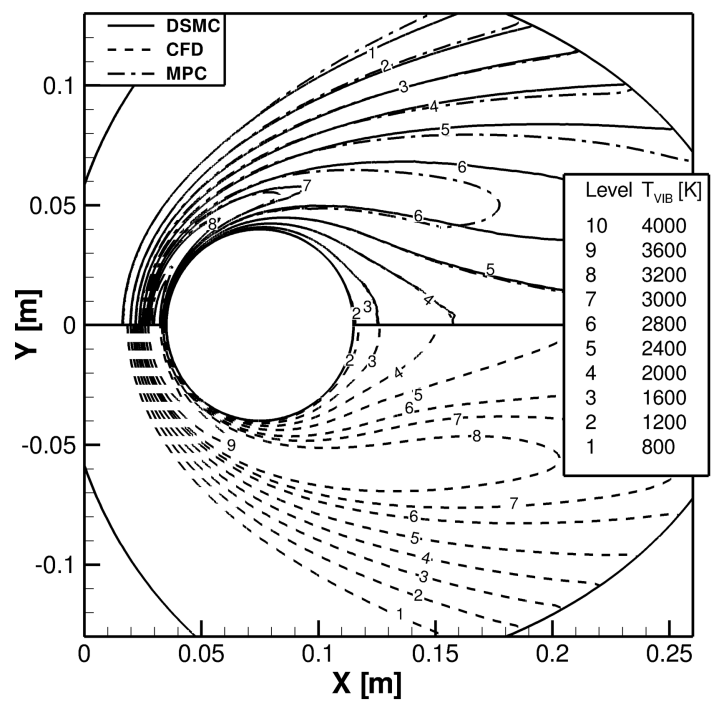

b) $T_{\mathrm{VIB}}$

Fig. 5 Comparison of temperature contours predicted by DSMC, CFD, and MPC methods for the $\mathrm{M}_{\mathrm{VP}}$ case.

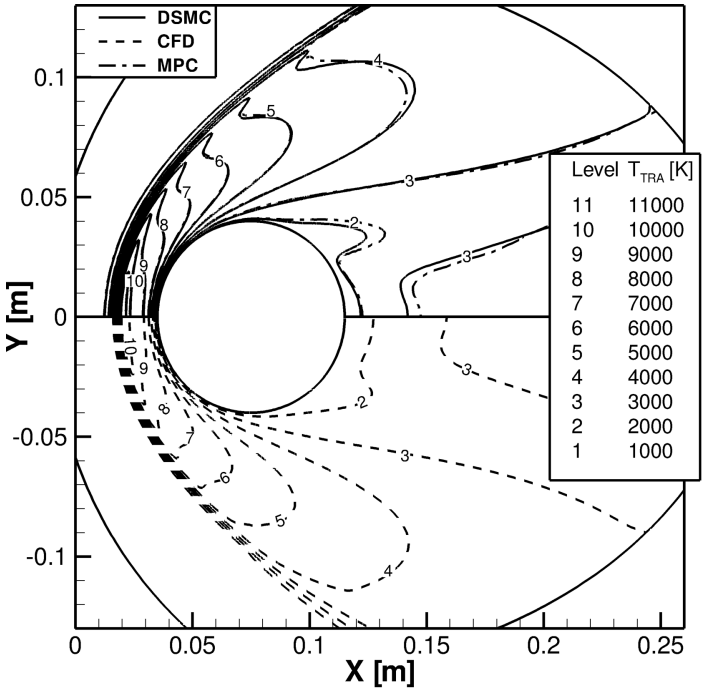

a) $T_{\text {TRA }}$

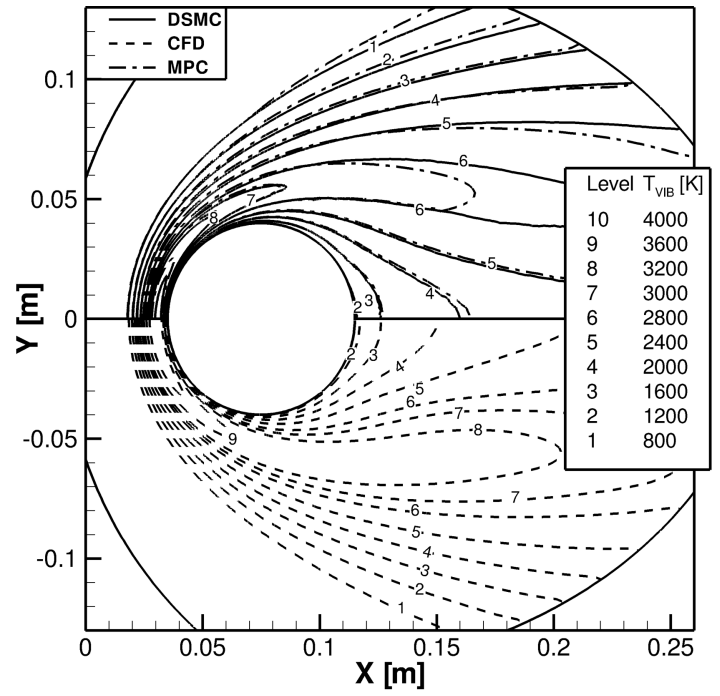

b) $T_{\mathrm{VIB}}$

Fig. 6 Comparison of temperature contours predicted by DSMC, CFD, and MPC methods for the $\mathrm{M}_{\mathrm{CB}}$ case.

the $\mathbf{M 1 5}_{\mathrm{VP}}$ and $\mathbf{M 1 5}_{\mathbf{C B}}$ cases, respectively. Similar to the vibrational temperature contour plots, the MPC method greatly improves agreement with full DSMC compared with full CFD. Despite the improved agreement, the effect of different relaxation rates in the M15 $_{\mathrm{VP}}$ is evident at the interface location between the CFD and DSMC modules. There is a distinct change in vibrational temperature gradient. This is caused by the difference in relaxation rates computed in each module. When compatible relaxation rates are used in the two modules, as is done for the $\mathbf{M 1 5}_{\mathbf{C B}}$ case, the solution is smoother with no abrupt change in gradient at the interface. Though small for this test case, differences in relaxation rates could have a larger effect on agreement between full DSMC and MPC methods for flows where the continuum region exhibits faster relaxation rates, which can occur in higher-enthalpy or higherdensity flows.

\section{B. Surface Properties}

For many hypersonic problems of interest, accurate prediction of surface properties has a strong impact on vehicle design. Predictions of heat transfer from DSMC, CFD, and MPC methods are compared. Equation (22) show the definitions of the heat transfer coefficient used to compare the surface properties predicted by the simulation

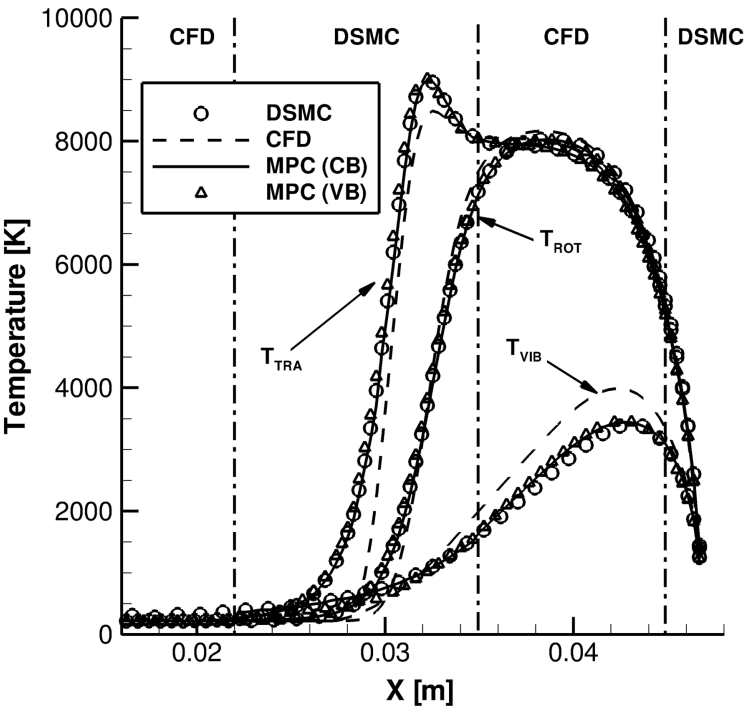

Fig. 7 Comparison of temperatures predictions by DSMC, CFD, and MPC methods along the $45^{\circ}$ extraction line for the $M 15_{\mathrm{VP}}$ and $M 15_{\mathrm{CB}}$ cases. 

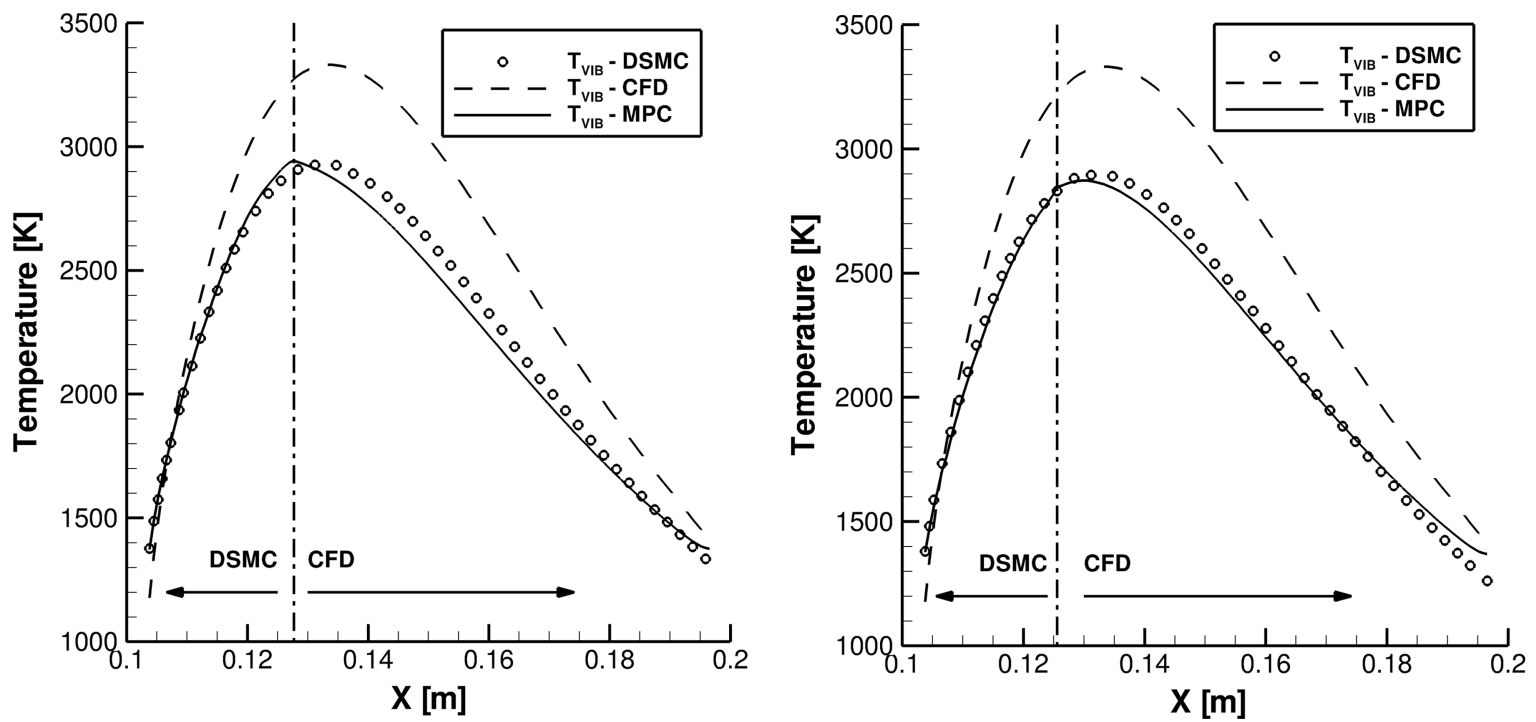

a) $\mathrm{M15}_{\mathrm{VP}}$

b) $\mathrm{M}_{\mathrm{CB}}$

Fig. 8 Comparison of vibrational temperature predicted by DSMC, CFD, and MPC methods along the $135^{\circ}$ extraction line for the $\mathrm{M}_{\mathrm{VP}}$ and $\mathrm{M}_{\mathrm{CB}}$ cases.

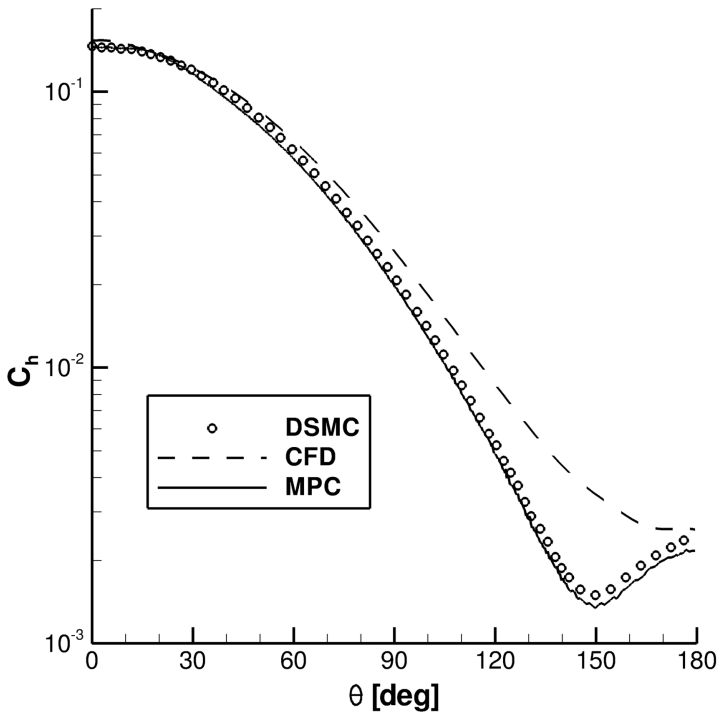

a) $\mathrm{M}_{\mathrm{VP}}$

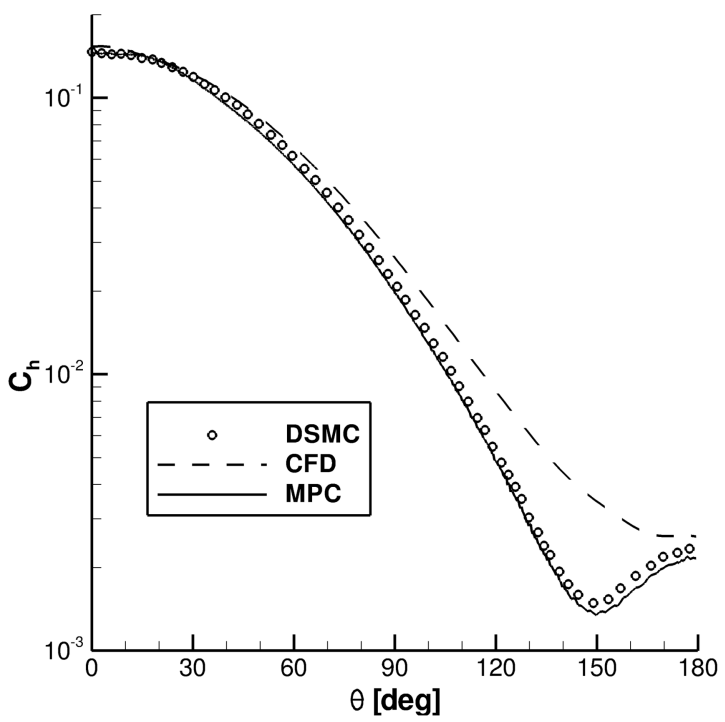

b) $\mathrm{M}_{\mathrm{CB}}$

Fig. 9 Comparison of coefficient of heat transfer along the cylinder surface predicted by DSMC, CFD, and MPC methods for the M15 ${ }_{\mathrm{VP}}$ and $\mathrm{M}_{\mathrm{CB}}$ cases.

methods where $q$ is the heat transfer to the wall, $\rho_{\infty}$ is the freestream density, and $V_{\infty}$ is the freestream velocity:

$$
C_{h}=\frac{q}{\frac{1}{2} \rho_{\infty} V_{\infty}^{3}}
$$

Figures $9 \mathrm{a}$ and $9 \mathrm{~b}$ show the heat transfer coefficient predicted by DSMC, CFD, and $\overline{M P C}$ methods for the $\mathbf{M 1 5}_{\mathrm{VP}}$ and $\mathbf{M} 15_{\mathrm{CB}}$ cases, respectively. Despite the slightly improved agreement in the prediction of vibrational temperature between DSMC and MPC methods for the $\mathbf{M 1 5} \mathbf{C B}_{\mathrm{CB}}$, both cases have the same level of agreement between the MPC method and full DSMC in heat transfer to the body. This is due to both MPC results being in excellent agreement with full DSMC near the body for both cases. Again, the MPC method is able to reproduce full DSMC results, especially in the wake region, where CFD overpredicts the heat transfer to the body by a factor of 3 .

\section{Computational Performance}

The MPC method reproduces full DSMC results by using DSMC in rarefied regions while decreasing the computational cost by limiting DSMC to only regions that are in translational nonequilibrium. Continuum regions are computed using an implicit Navier-Stokes solver. By loosely coupling the methods and studying steady-state flows, the time step used by CFD can be over 100 times larger than the time step required by DSMC for the corresponding flow. In addition, decoupling of the mesh densities allows CFD to be unrestricted by DSMC cell size restrictions, and CFD can use cells that are up to 18 times larger in area than DSMC. This significantly decreases the computational time and memory usage required by the MPC method to reproduce full DSMC results. The decreases in computational cost of the MPC method compared with full DSMC are summarized in Table 1. To make a fair comparison, the number of sample time steps after reaching steady state are the same for both full DSMC and MPC DSMC modules. Here, the actual speedup is defined as the ratio of the time required for the full DSMC calculation 
Table 1 Computational performance and memory requirements for the MPC method

\begin{tabular}{lccc}
\hline \hline Case & Actual speedup & Ideal speedup & Memory usage \\
\hline M15 $_{\text {VP }}$ & 3.37 & 3.07 & $22 \%$ \\
M15 $_{\text {CB }}$ & 3.96 & 3.16 & $27 \%$ \\
\hline \hline
\end{tabular}

to the time required for the MPC method calculation. The ideal speedup is defined as the ratio of particles used in a full DSMC simulation compared with the number in the corresponding MPC simulation. Since the computational cost of a DSMC simulation nearly scales linearly with the number of particles in the simulation, this should be the upper limit for computational speedup for that problem. The MPC method actually outperforms the ideal speedup ratio. This is because the required number of unsteady iterations in the MPC computation is reduced compared with the corresponding DSMC simulation, since MPC simulations begin with a fully converged CFD solution. The memory usage is defined as the ratio of the memory used by an MPC simulation to the memory used by the corresponding DSMC simulation. Here, the MPC method requires significantly less memory compared with full DSMC. The actual speedup for the cell-based relaxation case significantly outperforms the variable probability case. This is, in part, due to a slightly smaller DSMC region, but mostly can be attributed to a decrease in the number of iterations required to reach steady state, since the initial CFD solution that the MPC method starts with is closer to the final full DSMC solution.

\section{Conclusions}

The implementation of vibrational relaxation within a modular particle-continuum (MPC) method was described and tested on a hypersonic, blunt-body flow where small regions of the flow exhibited translational nonequilibrium. An MPC method has been outlined that uses existing DSMC and CFD codes with very little modification as modules within a hybrid code. This implementation allows separate updates of either the DSMC or CFD source codes, which reduces the development time of the hybrid code as newer physical models are added to each code. Information-transfer techniques of flow variables between DSMC and CFD modules were extended to account for vibrational energy. A method for controlling the statistical scatter of vibrational temperature was described and tested. This enables the MPC method to handle information transfer, including vibrational energy, in an accurate and efficient manner. Comparison of the effect of the vibrational-relaxation model used in the DSMC module was performed. It was found that a slight improvement in agreement of vibrational temperatures was possible, but did not have an effect on surface properties. With the inclusion of added physical models, such as finite rate chemistry, these improvements could have a larger effect on surface properties.

Comparison of near-equilibrium flowfields predicted by full DSMC, full CFD, and MPC methods showed that by only using DSMC in regions that are rarefied, the MPC method was able to reproduce full DSMC results while reducing the computational expense. It was found that using more consistent physical models also decreased the computational requirements of the MPC method, since the initial CFD solution was closer to the final nonequilibrium solution.

\section{Acknowledgment}

The authors gratefully acknowledge the financial support provided by NASA grant NCC3-989.

\section{References}

[1] Bird, G. A., Molecular Gas Dynamics and the Direct Simulation of Gas Flows, Clarendon Press, Oxford, 1994.

[2] Glass, C. E., and Gnoffo, P. A., "A 3-D Coupled CFD-DSMC Solution Method With Application to the Mars Sample Return Orbiter," NASA TM-2000-210322, July 2000.
[3] Glass, C. E., and Gnoffo, P. A., "Comparison of a 3-D CFD-DSMC Soultion Methodology With a Wind Tunnel Experiment," NASA TM2002-211777, Aug. 2002.

[4] Wilmoth, R. G., Mitcheltree, R. A., Moss, J. N., and K. D. V., "Zonally Decoupled Direct Simulation Monte Carlo Solutions of Hypersonic Blunt-Body Flows," Journal of Spacecraft and Rockets, Vol. 31, No. 6, 1994, pp. 971-979. doi: $10.2514 / 3.26546$

[5] Hash, D. B., and Hassan, H. A., "Assessment of Schemes for Coupling Monte Carlo and Navier-Stokes Solution Methods," Journal of Thermophysics and Heat Transfer, Vol. 10, No. 2, 1996, pp. 242-249. doi: $10.2514 / 3.781$

[6] Hash, D. B., and Hassan, H. A., "A Decoupled DSMC/Navier-Stokes Analysis of a Transitional Flow Experiment," AIAA Paper 1996-353, 1996.

[7] Roveda, R., Goldstein, D. B., and Varghese, P. L., "Hybrid Euler/ Particle Approach for Continuum/Rarefied Flows," Journal of Spacecraft and Rockets, Vol. 35, No. 3, 1998, pp. 258-265. doi: $10.2514 / 2.3349$

[8] Roveda, R., Goldstein, D. B., and Varghese, P. L., "Hybrid Euler/Direct Simulation Monte Carlo of Unsteady Slit Flow," Journal of Spacecraft and Rockets, Vol. 37, No. 6, 2000, pp. 753-760. doi: $10.2514 / 2.3647$

[9] Schwartzentruber, T. E., and Boyd, I. D., "A Hybrid ParticleContinuum Method Applied to Shock Waves," Journal of Computational Physics, Vol. 215, No. 2, July 2006, pp. 402-416. doi:10.1016/j.jcp.2005.10.023

[10] Schwartzentruber, T. E., Scalabrin, L. C., and Boyd, I. D., "Hybrid Particle-Continuum Simulations of Nonequilibrium Hypersonic BluntBody Flowfields," Journal of Thermophysics and Heat Transfer, Vol. 22, No. 1, 2008, pp. 29-37. doi:10.2514/1.30216

[11] Schwartzentruber, T. E., Scalabrin, L. C., and Boyd, I. D., "Multiscale Particle-Continuum Simulations of Low Knudsen Number Hypersonic Flow over a Planetary Probe," Journal of Spacecraft and Rockets, Vol. 45, No. 6, 2008, pp. 1196-1206. doi:10.2514/1.37319

[12] Schwartzentruber, T. E., Scalabrin, L. C., and Boyd, I. D., "Modular Implementation of a Hybrid DSMC-NS Algorithm for Hypersonic Non-Equilibrium Flows," AIAA Paper 2007-613, 2007.

[13] Deschenes, T. R., Holman, T. D., and Boyd, I. D., "Effects of Rotational Energy Relaxation in a Modular Particle-Continuum Method," Journal of Thermophysics and Heat Transfer, Vol. 25, No. 2, 2011, pp. 218 227.

doi: $10.2514 / 1.50720$

[14] Schwartzentruber, T. E., Scalabrin, L. C., and Boyd, I. D., "A Modular Particle-Continuum Numerical Method for Hyper- Sonic NonEquilibrium Gas Flows," Journal of Computational Physics, Vol. 225, No. 1, July 2007, pp. 1159-1174. doi:10.1016/j.jcp.2007.01.022

[15] Scalabrin, L. C., and Boyd, I. D., "Numerical Simulation of Weakly Ionized Hypersonic Flow for Reentry Configurations," AIAA Paper 2006-3773, 2006.

[16] Scalabrin, L. C., and Boyd, I. D., "Development of an Unstructured Navier-Stokes Solver for Hypersonic Nonequilibrium Aerothermodynamics," AIAA Paper 2005-5203, June 2005.

[17] Dietrich, S., and Boyd, I. D., "Scalar and Parallel Optimized Implementation of the Direct Simulation Monte Carlo Method," Journal of Computational Physics, Vol. 126, No. 2, July 1996, pp. 328 342 . doi:10.1006/jcph.1996.0141

[18] Parker, J. G., "Rotational and Vibrational Relaxation in Diatomic Gases," Physics of Fluids, Vol. 2, No. 4, July 1959, pp. 449-462. doi:10.1063/1.1724417

[19] Holman, T. D., and Boyd, I. D., "Effects of Continuum Breakdown on the Surface Properties of a Hypersonic Sphere," Journal of Thermophysics and Heat Transfer, Vol. 23, No. 4, 2009, pp. 660-673. doi:10.2514/1.43509

[20] Boyd, I. D., "Analysis of Rotational Nonequilibrium in Standing Shock Waves of Nitrogen," AIAA Journal, Vol. 28, No. 11, 1990, pp. $1997-$ 1999. doi:10.2514/3.10511

[21] Millikan, R. C., and White, D. R., "Systematics of Vibrational Relaxation," Journal of Chemical Physics, Vol. 39, No. 12, Dec. 1963, pp. 3209-3213. doi:10.1063/1.1734182

[22] Park, C., Nonequilibrium Hypersonic Aerothermodynamics, Wiley, New York, 1990 
[23] Boyd, I. D., "Analysis of Vibrational-Translational Energy Transfer Using the Direct Simulation Monte Carlo Method," Physics of Fluids A, Vol. 3, No. 7, July 1991, pp. 1785-1791. doi:10.1063/1.857959

[24] Gimelshein, N. E., Gimelshein, S. F., and Levin, D. A., "Vibrational Relaxation Rates in the Direct Simulation Monte Carlo Method," Physics of Fluids, Vol. 14, No. 12, 2002, pp. 4452-4455. doi:10.1063/1.1517297

[25] Lofthouse, A. J., Scalabrin, L. C., and Boyd, I. D., "Hypersonic Aerothermodynamics Analysis Across Nonequilibrium Regimes Using Continuum and Particle Methods," AIAA Paper 2007-3903, 2007.

[26] Holman, T. D., "Numerical Investigation of the Effects of Continuum Breakdown on Hypersonic Vehicle Surface Properties by," Ph.D. Thesis, Univ. of Michigan, Ann Arbor, MI, 2010.

[27] Bergemann, F., and Boyd, I. D., "New Discrete Vibrational Energy Method for the Direct Simulation Monte Carlo Method," Rarefied Gas Dynamics, Progress in Astronautics and Aeronautics, AIAA, Washington, D.C., 1994, pp. 174-180.

[28] Boyd, I. D., Chen, G., and Candler, G. V., "Predicting Failure of the
Continuum Fluid Equations in Transitional Hypersonic Flows," Physics of Fluids, Vol. 7, No. 1, Jan. 1995, pp. 210-219. doi: $10.1063 / 1.868720$

[29] Wang, W.-L., and Boyd, I. D., "Predicting Continuum Breakdown in Hypersonic Viscous Flows," Physics of Fluids, Vol. 15, No. 1, Jan. 2003, pp. 91-100. doi:10.1063/1.1524183

[30] Schwartzentruber, T. E., Scalabrin, L. C., and Boyd, I. D., "Investigation of Continuum Breakdown in Hypersonic Flows using a Hybrid Particle-Continuum Algorithm," AIAA Paper 2008-4108, 2008.

[31] Sun, Q., and Boyd, I. D., "Evaluation of Macroscopic Properties in the Direct Simulation Method," Journal of Thermophysics and Heat Transfer, Vol. 19, No. 3, 2005, pp. 329-335. doi: $10.2514 / 1.12542$

X. Zhong Associate Editor 